\title{
La Fonction esthétique du paratexte gidien
}

\author{
David Keypour
}

Huron College, Université de Western Ontario, London

\begin{abstract}
T e propos de cet article est d'examiner tout d'abord certaines prises de position théoriques de Gérard Genette dans son livre sur le paratexte, ${ }^{1}$ d'isoler ensuite les éléments les plus admissibles, à mon sens, au titre de paratextualité des Faux-Monnayeurs et enfin, d'analyser l'effet particulier de ces éléments sur le plan esthétique, plan qui me semble le plus pertinent et que Genette est amené à négliger à cause de la restriction qu'il impose aux fonctions multiples de l'appareil paratextuel.
\end{abstract}

\section{I}

D'après Genette, le paratexte est le discours extra-diégétique, oral ou écrit, que l'auteur ou des personnes accréditées par lui ont tenu sur son texte diégétique. Ce discours se caractérise par sa position spatiale hors-texte, et par son occurrence temporelle principalement avec le texte, mais pouvant se produire également avant ou après la première publication du texte. Le paratexte constitue "le versant le plus socialisé de la pratique littéraire" (p. 18), car c'est un ensemble de dispositifs discursifs qui, intentionnellement ou incidemment, affecte la réception du texte littéraire et en oriente la lecture. Cet effet pragmatique étant, selon Genette, la raison d'être du paratexte, il n'est pas étonnant qu'il en fasse également l'objet principal de sa recherche. C'est ce qui le conduit à détecter sous l'innocence apparente ou réelle de chaque élément de paratexte le partage des responsabilités entre l'auteur, son éditeur et d'autres instances qui au su ou à l'insu de lauteur participent à la production d'une certaine image de l'oeuvre. D'autre part, l'étude de Genette étant un essai de formalisation a priori du statut, des caractéristiques et des fonctions de chaque élément paratextuel, formalisation opérée sur "une grille générale et constante de possibles alternatifs" (p. 17), elle s'interdit d'aborder "les effets de convergence ou de divergence qui rêsultent de la composition, autour du texte, de l'ensemble de son paratexte" (p. 17); encore moins se propose-t-elle de 
faire une telle synthèse pour quelque oeuvre en particulier. Et pour illustrer ses propos théoriques, Genette choisit souvent ses exemples parmi les paratextes gidiens et se réfère parfois exclusivement au paratexte des Faux-Monnayeurs, ce qui a également déterminé mon choix exclusif de l'oeuvre gidienne.

Genette ne manque pas d'insister sur les difficultés que l'on rencontre à identifier et à délimiter avec précision les composantes du paratexte. Même sur le plan spatio-temporel, dit-il dans son introduction, "plus que d'une limite ou d'une frontière étanche, il s'agit ici d'un seuil [...], [d']une "Zone indécise" entre le dedans et le dehors, elle-même sans limite rigoureuse, ni vers l'intérieur (le texte) ni vers l'extérieur (le discours du monde sur le texte)" (p. 7-8).

Il convient de relever tout d'abord la suggestion qui est faite ici d'une symétrie égalitaire entre les deux "zones indécises" qui entourent de part et d'autre le champ paratextuel tel que le délimitera Genette dans le corps de son étude. En effet, il est évident que, dans son mouvement vers l'intérieur du texte, le paratexte rencontre un "seuil" qui est le premier mot du récit posé très précisément dans l'espace du livre et dans le temps de la lecture. Par ailleurs, le paratexte n'a que la possibilité théorique de franchir ce seuil, et quand il le franchit, il se limite à deux éléments. Car les seuls éléments du paratexte qui peuvent éventuellement figurer dans l'espace de l'oeuvre sont les inter-titres et les épigraphes de chapitres.

Or dans son mouvement vers l'extérieur, vers ce que Genette appelle "le discours du monde sur le texte," le paratexte Genettien ne rencontre aucun "seuil" ni dans l'espace, ni dans le temps, puisque les dimensions de ce "discours sur le texte" peuvent embrasser toute la "République des lettres" (p. 18) dans son étendue et dans sa durée. Genette va même plus loin dans ce sens lorsqu'il inclut dans le champ du paratexte des éléments qu'il qualifie de factuels et qui consistent en des messages non pas explicites mais en des faits "dont la seule existence, si elle est connue du public, apporte quelque commentaire au texte et pèse sur sa réception [...] ainsi de l'âge ou du sexe de lauteur [...] ou de la date de l'oeuvre [...] l'appartenance à une académie [...] ou l'obtention d'un prix littéraire» (p. 12-13). Ou plus fondamentalement encore, l'inscription d'un livre dans la production globale de son auteur ou au sein d'un genre déterminé ou d'une période historique. Bref, dit Genette, "tout contexte fait paratexte" (p. 13).

Par ailleurs cependant, Genette prévient contre la tendance à soutenir que tout est paratexte et il limite volontairement son travail au paratexte verbalement explicité. De plus il ne considère comme paratexte que ce qui est commandé par "une intention et une responsabilitê de l'auteur" (p. 9). Ainsi le champ du paratexte 
est divisé en "péritexte," comprenant les éléments qui accompagnent le volume de l'oeuvre littéraire ou qui figurent entre ses deux couvertures, et en "épitexte," comprenant tout discours extérieur au volume mais pouvant l'éclairer d'une certaine manière. Cependant, est éliminé de l'épitexte tout discours critique, qu'il soit journalistique ou savant bien que maints lecteurs fassent la connaissance d'un livre à la faveur d'un compte rendu journalistique et que leur lecture soit affectée par ce qu'ils ont pu lire au sujet de l'oeuvre en question ou des oeuvres antérieures de son auteur. Par contre, il inclut dans le champ du paratexte les déclarations de l'auteur, qu'il les ait confiées à son journal, à ses intimes qui les auraient recueillies et publiées ou à des interviewers éventuels. Ainsi en ce qui concerne Gide par exemple, Genette donne comme faisant partie de l'épitexte, le Journal, le Journal des Faux-Monnayeurs, la Correspondance, mais aussi les Cahiers de la Petite Dame, le Gide familier de Jean Lambert, Une mort ambiguë de Robert Mallet, les Notes sur André Gide de Roger Martin Du Gard et les Entretiens avec Jean Amrouche.

La question que soulève une telle sélection est celle de son caractère arbitraire. Car il saute aux yeux que le champ paratextuel gidien ainsi défini est ou bien trop restreint ou bien trop étendu. Car la plupart de ces livres esquissent plutôt des portraits de Gide en tant qu'homme et ne jettent qu'une faible lumière et involontaire, de quelque nuance soit-elle, sur l'oeuvre de l'auteur. Dans le sens contraire, il suffit de mettre en regard des titres ci-dessus mentionnés Jeunesse d'André Gide de Jean Delay pour se rendre compte de l'insuffisance des choix de Genette. En réalité, ce choix est révélateur d'un parti-pris ambigu de la part du théoricien. C'est que la définition genettienne du paratexte se fonde à la fois sur une approche textologique et sur une approche relevant de l'économique, plus précisément, de cette branche de l'économie qui s'occupe du marketing de l'industrie du livre. En effet, le concept qui sert de norme à la définition de la paratextualité et qui est celle de réception du texte littéraire, renferme deux notions distinctes: d'une part "réception" au sens d'accueil de la part du public, de nombre d'exemplaires vendus, de succès commercial donc, d'autre part au sens d'interprétation, de mode de comprêhension, d'image du livre que le paratexte est susceptible de conditionner chez le lecteur. Et paradoxalement, c'est la première notion, celle de laccuell qui semble dominer l'autre, même quand Genette s'attache à analyser le statut et la pragmatique d'éléments aussi textuels que le titre de l'oeuvre, sa dédicace ou ses épigraphes. C'est aussi ce qui explique l'élimination de toute critique surtout la critique dite universitaire ou savante, et en ce qui concerne le paratexte de Gide, la prise en compte du Gide familier de Lambert, livre que chacun peut lire dans le 
métro, et l'exclusion de Jeunesse d'André Gide, ouvrage pourtant nourri de vastes extraits de correspondance du jeune Gide, mais trop gros et trop riche de substance pour la masse des lecteurs qui font le succès commercial des romans. Au demeurant, Genette ne se contredit point, il pousse jusqu'à ses extrêmes conséquences le projet qui est le sien, à savoir, l'étude des dispositifs discursifs et factuels qui affectent "le versant le plus socialisés de la pratique littéraire," et s'il avait pu prévoir la fatwa contre les Versets sataniques de Salman Rushdie, il aurait cité la condamnation à mort par quelqu'Ayatollah, à côté des prix littéraires, comme facteur essentiel de la réception du texte.

\section{II}

Je voudrais proposer pour ma part une définition de la réception du texte littéraire telle qu'elle écarte toute notion de succès et de consommation et qu'elle n'en retienne que le sens purement interprétatif, celui qui a rapport à l'orientation de la lecture du texte. Appartiendrait alors au champ du paratexte tout élément exclusivement discursif qui émane directement de lauteur et qui accompagne d'une manière permanente le texte littéraire dans le volume même qui le contient ou qui s'y rattache par des liens que je qualifierais de syntaxiquement nécessaires. De tels éléments, ne se laissant découvrir que par la lecture de l'oeuvre même, ou par leur présence à son horizon immédiat mais permanent, constitueront un système discursif intégré qui portant sur le système premier du texte est proprement un métalangage. Il s'agira alors d'examiner justement ce que l'approche de Genette ne permet pas d'examiner, c'est-à-dire, "les effets de convergence ou de divergence qui résultent de la composition, autour du texte, de [cet] ensemble" (p. 17), d'analyser, autrement dit, les effets esthétiques qui rêsultent des rapports entre le texte et ce paratexte. Conformément à la norme que nous venons de poser le paratexte des Faux-Monnayeurs se limiterait aux éléments suivants: le titre, les inter-titres, la dédicace, les épigraphes et le Journal des Faux-Monnayeurs. Mais est-ce tout?

Dans un article intitulé "Texte littéraire et métalangage," ${ }^{2}$ Philippe Hamon souligne que contrairement à la communication orale personnalisée et contextualisée par nature, le texte littéraire constitue un mode de "communication différée" donc "décontextualisée, hermétique et ambiguë que l'on peut définir comme un carrefour d'absences et de malentendus labsence de l'émetteur et du contexte 
d'émission pour le récepteur, absence symétrique du récepteur et du contexte de réception pour l'émetteur, etc.)" (p. 264). D'où "l'hypothèse que le texte littéraire, non seulement est générateur de paraphrases, de gloses, d'explications, d'exégèses [...] et de rewritings extérieurs divers, mais qu'il contient son propre système de paraphrases, son propre métalangage interne, et qu'il pourrait se définir, à la limite, comme un énoncé à métalangage incorporéx (p. 265). Ce métalangage est éclaté en "faits et procédés stylistiques disparates et hétẻroclites [...] qui] peuvent s'incarner dans des unités textuelles de grandeur et de nature très diverses (un mot ou groupe de mots, une paraphrase de détail, une "structure," comme au niveau d'une séquence narrative étendue, voire du texte tout entier) et jouant sur des plans très divers (au niveau de l'énoncé comme de l'énonciation)." Mais ces éclats de métalangage "tendent à se concentrer à des endroits privilégiés du texte" (p. 265) dont les plus évidents sont le cadre du texte et les frontières entre ses diverses sêquences. Et Philippe Hamon mentionne expressément, au début des textes, les titres, préfaces, frontispices, avis au lecteur, indicateurs de genre, exergues, et à la fin des textes, conclusions, péroraisons, tables des matières, etc., fonctionnant tous "comme des condensés (ou des maquettes) de fragments [...] du texte [...] ou du texte tout entier, introduisant des horizons d'attente prospectifs ou des bilans rêcapitulatifs, en proposant des "mises en équivalence" du texte avec lui-même" (p. 267).

On voit clairement que les exemples mentionnés par Hamon englobent les éléments du paratexte gidien que nous avons retenus. Mais le plus important de notre point de vue c'est que son analyse théorique définit le paratexte comme un métalangage organiquement lié au système du texte littéraire et qu'elle autorise à repérer ailleurs qu'aux frontières externes et internes de ce texte des séquences qui pourralent légitimement faire partie du paratexte. Ainsi, en ce qui concerne Les Fawx-Monnayeurs, il semble permis de compter parmi de telles séquences le journal d'Edouard, la discussion sur le roman à Saas-Fée dans la seconde partie du roman et le chapitre 7 de cette même partie où le romancier médite explicitement sur ses personnages, et le Journal des Faux-Monnayeurs que nous avons déjà mentionné. L'appareil paratextuel étant ainsi constitué, il s'agit d'en étudier les rapports avec le texte. 


\section{III}

Je laisse de côté la dédicace à Roger Martin Du Gard sauf pour relever non pas les implications du terme "roman" qui s'y trouve, mais linsistance sur la propriété de l'oeuvre, insistance que l'on peut détecter sous l'emplol du possessif "mon premier roman" si l'on veut bien admettre que d'autres alternatives étaient possibles, y compris celle d'une dédicace sans indication de genre et réduite à: "A ROGER MARTIN DU GARD en témoignage d'amitié," le mot "roman" isolé de la dédicace pouvant former sous-titre à valeur générique comme de coutume.

Ce soupçon qui parait aussi absurde que l'insistance éventuelle de l'auteur gagne quelque légitimité quand on s'interroge sur le titre: de qui ou de quoi s'agit-il? Le titre désigne-t-il les personnages ou quelques-uns des personnages du roman ou désigne-t-il le roman lui-même, comme entité littéraire? Mais ce roman ne raconte-t-il pas l'aventure du roman d'Edouard intitulé Les Fawx-Monnayeurs autant qu'il raconte l'histoire des personnages? Et le titre ne viserait-il pas ce roman comme L'Éducation sentimentale, par exemple, vise les projets amoureux de Frédéric Moreau, ou mieux encore, comme le titre du Chef d'oeuvre inconnu de Balzac vise le tableau du vieux maitre Frenhofer? Quelle que soit notre lecture du titre, il est impossible d'échapper à l'effet spéculaire qui se produit grâce à l'identité des deux titres; et puisqu'il y a spécularité, l'on en vient à se demander si le titre du roman d'Edouard ne désigne pas celui de Gide. Le possessif de la dédicace ne serait-il pas dans ce cas motivé par ce confus sentiment de rivalité entre le roman de Gide et celui d'Édouard son double?

Cette spécularité transparaît également dans le jeu des inter-titres des trois parties du roman: "Paris," "Saas-Fée," "Paris." Désignations purement géographiques vides de tout contenu narratif. Le métalangage ici s'abstient de remplir sa fonction sémantique pour se poser comme signe d'une symétrie de part et d'autre - ou est-ce au-dedans et au-dehors? - d'un miroir. Or Saas-Fée plan de symétrie entre les deux séries d'aventures parisiennes des personnages est aussi le miroir de la gestation des deux romans rivaux; car c'est là, sur les hauteurs de Saas-Fée qu'Edouard expose son projet de roman et s'empêtre dans ses explications, et c'est là aussi que, au chapitre 7 , comme un rvoyageur, parvenu au haut de la colline [...] l'auteur imprévoyant s'arrête un instant, reprend souffle, et se demande avec inquiêtude où va le mener son récit" (p. 1108). ${ }^{3}$ Faut-il voir dans la position de ce chapitre après l'exposé d'Edouard où ce dernier enfin révèle le titre de son roman, 
une tentative de la part de l'auteur d'affirmer son autorité malgré l'identité des titres et la similitude des projets?

Mais cette autorité est déjà gravement contestée par le jeu des êpigraphes. Il est évident que l'épigraphe, pur métalangage, citation par excellence et ne pouvant exister de surcroît que sous forme écrite, est essentiellement hétérogène aux discours des personnages comme à celui du narrateur, qu'elle est cueillie par l'auteur et transplantée par lui de manière à condenser sa pensée sous-jacente à l'intrigue toute entière ou à tel épisode du roman qu'elle vient coiffer. Il est donc de la plus grande importance que, si une épigraphe précède une séquence du journal d'Edouard, cette épigraphe soit posée en dehors de l'espace typographique occupé par le texte du journal. Or, bien que ce soit souvent le cas, il arrive que l'épigraphe figure en tête d'un passage du journal après la présentation du passage par la mention "Journal d'Edouard," comme si l'épigraphe en faisait partie. Il y a plus grave empiètement sur l'autorité de Gide lorsqu'Edouard choisit une épigraphe chez Paul Bourget: "La famille..., cette cellule sociale." pour un chapitre de son roman et qu'il se trouve que cette épigraphe condense mieux que toute autre la thématique profonde de tout le roman de Gide.

Ainsi, Edouard écrivant son journal, semble subrepticement se substituer à l'instance de l'écriture jusqu'à régir la voix du narrateur. Cela se constate également dans le système de datation de ce journal. En effet, Edouard n'inscrit les dates des entrées de son journal que lorsqu'elles sont nécessaires à la clarté chronologique des événements rapportés par le narrateur comme s'il savait ce que ce dernier raconte. L'inscription d'une de ces dates est particulièrement troublante en ce qu'elle marque nettement le renversement de la hiérarchie des instances narratives: auteur, narrateur, personnages. Il s'agit encore d'une séquence de la seconde partie (où semble décidément se concentrer la rivalité entre les instances), et justement après la grande discussion. Cet épisode est raconté par le narrateur; mais lorsqu'après coup Edouard prend la plume pour écrire les réflexions que l'épisode lui inspire, au lieu de poser la date, il inscrit "Ce même soir." Le démonstratif "ce" transforme le discours du narrateur en l'objet du métalangage d'Edouard.

Mais c'est tout le roman qui se révèle être l'objet de ce métalangage lorsqu'on porte l'attention à l'endroit peut-être le plus stratégique de tout roman, c'est-à-dire à sa fin, et que l'on constate que ce n'est pas l'auteur, ni le narrateur qui mettent fin au discours narratif, mais c'est Edouard qui en interrompant son journal, interrompt le récit. 
Enfin on peut se demander pourquoi Gide a tenu à publier séparément le Journal des Faux-Monnayeurs alors qu'il en avait bel et bien versé l'essentiel dans le roman même, "pour le plus grand embarras du lecteur," comme il l'a écrit.

Il est commun de nos jours, dans le monde du cinéma surtout, de produire des séquelles à une première réussite. Or ces séquelles, signes de succès sur le plan de réception au sens de "l'accueil," s'analysent sur le plan de réception au sens linguistique comme signes de la fonction que Jakobson appelle la "fonction phatique du langage." Le cinéaste continuant d'affirmer sa présence au monde en relançant son premier message par des rappels. Il y a de cela dans la publication du Journal des Faux-Monnayeurs. Mais surtout s'agissant d'un roman où l'auteur a tenté de représenter à côté d'une fiction l'histoire de sa tentative même, cette histoire est devenue l'histoire fictive du roman fictif d'Edouard, puisque rien ne peut entrer dans un roman sans basculer immédiatement dans le fictif. La fonction sous-jacente à toutes les fonctions d'un métalangage est d'affirmer la propriété ou l'appropriation du langage premier, et si malgré tous ces jeux, l'écriture des Faux-Monnayeurs témoigne du drame de la dépossession du langage au moment même où on l'exerce, le Journal des Faux-Monnayeurs a la valeur d'un titre de propriêté, d'une tentative de saisie sur cette parole qui se parle à travers le créateur et qui semble échapper à son autorité.

\section{IV}

Dans une analyse des rapports entre le journal intime et le récit, Maurice Blanchot écrit: "Il semble que doivent rester incommunicables l'expérience propre de l'oeuvre, la vision par laquelle elle commence, l'espèce d'égarement qu'elle provoque, et les rapports insolites qu'elle établit entre l'homme que nous pouvons rencontrer chaque jour et qui précisément tient journal de lui-même et cet être que nous voyons se lever derrière chaque grande oeuvre. ${ }^{4}$ Gide n'aurait-il pas vécu cette espèce d'égarement, ses rapports insolites, lui qui a écrit cette phrase proprement fantastique: "Il est certain que si Je, romancier, porte en moi le personnage d'Edouard, je dois porter également le roman qu'il écrit" comme s'll était plus convaincu de la réalité d'Edouard que de la sienne propre, de la réalité du roman d'Edouard que de celle du sien, et qu'il cherchait à se rassurer par analogie avec son personnage? 


\section{Notes}

${ }^{1}$ Gérard Genette, Seuils (Paris: Editions du Seuil, 1987).

${ }^{2}$ Critique nos 29-32 (1977).

${ }^{3}$ Edition de la Pléiade.

${ }^{4}$ Le Livre à venir, p. 229. 